\title{
M Channel KCNQ2 Subunits Are Localized to Key Sites for Control of Neuronal Network Oscillations and Synchronization in Mouse Brain
}

\author{
Edward C. Cooper, ${ }^{1,2}$ Emily Harrington, ${ }^{1}$ Yuh Nung Jan, ${ }^{2,3,4}$ and Lily Y. Jann ${ }^{2,3,4}$ \\ Departments of ${ }^{1}$ Neurology, ${ }^{2}$ Physiology, and ${ }^{3}$ Biochemistry and ${ }^{4}$ Howard Hughes Medical Institute, University of \\ California, San Francisco, San Francisco, California 94143-0725
}

Mutations in the potassium channel subunit KCNQ2 lead to benign familial neonatal convulsions, a dominantly inherited form of generalized epilepsy. In heterologous cells, KCNQ2 expression yields voltage-gated potassium channels that activate slowly $(\tau, \sim 0.1 \mathrm{sec})$ at subthreshold membrane potentials. KCNQ2 associates with KCNQ3, a homolog, to form heteromeric channels responsible for the $M$ current $\left(I_{M}\right)$ in superior cervical ganglion (SCG) neurons. Muscarinic acetylcholine and peptidergic receptors inhibit SCG $I_{M}$, causing slow EPSPs and enhancing excitability. Here, we use KCNQ2N antibodies, directed against a conserved $\mathrm{N}$-terminal portion of the KCNQ2 polypeptide, to localize KCNQ2-containing channels throughout mouse brain. We show that KCNQ2N immunoreactivity, although widespread, is particularly concentrated at key sites for control of rhythmic neuronal activity and synchronization. In the basal ganglia, we find KCNQ2N immunoreactivity on somata of dopaminergic and parvalbumin (PV)-positive (pre- sumed GABAergic) cells of the substantia nigra, cholinergic large aspiny neurons of the striatum, and GABAergic and cholinergic neurons of the globus pallidus. In the septum, GABAergic, purinergic, and cholinergic neurons that contribute to the septohippocampal and septohabenular pathways exhibit somatic KCNQ2 labeling. In the thalamus, GABAergic nucleus reticularis neurons that regulate thalamocortical oscillations show strong labeling. In the hippocampus, many PV-positive and additional PV-negative interneurons exhibit strong somatic staining, but labeling of pyramidal and dentate granule somata is weak. There is strong neuropil staining in many regions. In some instances, notably the hippocampal mossy fibers, evidence indicates this neuropil staining is presynaptic.

Key words: M current; acetylcholine; epilepsy; benign familial neonatal convulsions; channelopathy; neuromodulation; voltage-gated potassium channel
Genetic studies have revealed a new family of potassium channels called KCNQ channels that play key roles in heart, brain, and other tissues. Mutations in KCNQ1 cause hereditary long-QT syndrome, a cardiovascular disorder associated with syncope and sudden death (Wang et al., 1996). Although KCNQ1 has not been found in brain, investigators subsequently identified four homologous neuronal KCNQ genes (KCNQ2-5). KCNQ2 and KCNQ3 mutations cause benign familial neonatal convulsions (BFNC), a dominantly inherited epileptic syndrome characterized by seizures during infancy and in later life (Biervert et al., 1998; Singh et al., 1998). KCNQ4 mutations result in a form of dominantly inherited deafness (Kubisch et al., 1999). KCNQ5 is widely expressed in brain, heart, and skeletal muscle (Schroeder et al., 2000a).

Interactions between KCNQ subunits are important for channel properties but are incompletely understood. KCNQ1 interacts

\footnotetext{
Received July 30, 2001; revised Sept. 21, 2001; accepted Sept. 24, 2001.

This work was supported by an individual investigator grant from the University of California, San Francisco Academic Senate (E.C.C.), by National Institute of Neurological Disorders and Stroke Grant NS42100 (E.C.C.), and by a National Institute of Mental Health grant to the Silvio Conte Center for Neuroscience at University of California, San Francisco. L.Y.J. and Y.N.J. are investigators of the Howard Hughes Medical Institute. We thank T. Jentsch for human KCNQ2 and KCNQ3 cDNA clones, R. Edwards for anti-VAchT antibodies, S. Pleasure for use of the Leica microtome, and T. Surti for help with ChAT immunostaining.

Correspondence should be addressed to Dr. Edward C. Cooper, Departments of Neurology and Physiology, University of California, San Francisco, Room U232, 533 Parnassus Avenue, Box 0725, San Francisco, CA 94143-0725. E-mail: ecooper@ itsa.ucsf.edu.

Copyright (C) 2001 Society for Neuroscience $\quad 0270-6474 / 01 / 219529-12 \$ 15.00 / 0$
}

with the small accessory subunit KCNE1, forming heteromeric voltage-gated channels that activate much more slowly than those consisting of KCNQ1 alone (Sanguinetti et al., 1996). KCNQ1/ KCNE1 heteromers underlie a cardiac current, $I_{\mathrm{KS}}$, that helps repolarize $\mathrm{Ca}^{2+}$ action potentials. In contrast, association of KCNQ1 with another accessory protein, $\mathrm{KCNE3,} \mathrm{results} \mathrm{in}$ "leak" channels that are open at all physiological membrane potentials. Such channels are expressed in gastrointestinal epithelia, where they help regulate secretion (Schroeder et al., 2000b). KCNQ2 and KCNQ3 subunits associate in SCG neurons to underlie a slow, voltage-gated potassium current, $I_{\mathrm{M}}$ (Brown and Adams, 1980; Wang et al., 1998). BFNC may be attributable to impaired activity of central KCNQ2/KCNQ3 heteromers, because disease mutations in either subunit reduce current density (Schroeder et al., 1998), and the two subunits can be reciprocally coimmunoprecipitated from human brain (Cooper et al., 2000).

Although KCNQ2/KCNQ3 heteromers are likely expressed by both central and sympathetic neurons, understanding of the subunit composition and properties of neuronal KCNQ channels is limited. KCNQ2, KCNQ3, and KCNQ5 mRNAs exhibit wide, mostly overlapping distribution, whereas KCNQ4 mRNA and protein appear concentrated in the cochlea and a few brainstem nuclei (Kharkovets et al., 2000). Unlike KCNQ2, KCNQ3 can form functional heteromers with KCNQ4 or KCNQ5 in heterologous cells (Kubisch et al., 1999; Schroeder et al., 2000a). It is not known whether these combinations are found in vivo. Whether the properties of neuronal KCNQ channels are modified by accessory subunits is also unknown. Many alternatively spliced 
KCNQ2 mRNA isoforms have been isolated from brain and sympathetic ganglia (Nakamura et al., 1998). When expressed heterologously, the products of these mRNAs differ in their ability to form functional channels alone, interactions with KCNQ3, and kinetics (Pan et al., 2001; Smith et al., 2001).

The association of epilepsy with KCNQ2 mutations that cause only small reductions in channel activity (Schroeder et al., 1998) prompted us to attempt to identify the central neurons and circuits that appear so exquisitely sensitive to KCNQ channel levels. We mapped KCNQ2 immunoreactivity in brain sections using an antibody directed against a region of KCNQ2 conserved among known splice variants. We found that KCNQ2 immunoreactivity, although widespread, is concentrated at key locations for control of brain rhythmic activity and neuronal synchronization.

\section{MATERIALS AND METHODS}

Materials. Plasmids encoding human KCNQ2 and KCNQ3 were gifts from Drs. T. Jentsch and B. Schroeder (Zentrum fur Moleculare Neurobiologie, Hamburg, Germany); rabbit anti-vesicular acetylcholine transporter (VAchT) antibodies were a gift from Dr. Robert Edwards (University of California, San Francisco, CA). Donkey anti-rabbit HRPconjugated antibodies (Amersham Pharmacia Biotech, Arlington Heights, IL) were used for Western blots. Dr. Christoph Turck (Howard Hughes Medical Institute, University of California, San Francisco) synthesized the KCNQ2N peptide.

Generation of anti-KCNQ2N antibodies. A peptide sequence derived from the N-terminal region of KCNQ2N (GEKKLK VGFVGLDPGAPDSTRDC) was selected after analysis using MacVector software indicated high immunogenicity, and BLAST database searches showed that the sequence was unique to KCNQ2 and conserved among all known KCNQ2 clones. The peptide was conjugated to keyhole limpet hemocyanin via a cysteine residue added at the $\mathrm{C}$ terminus of the peptide during synthesis. Two rabbits were immunized, and sera were collected (Animal Pharm Services, Healdsburg, CA). After screening experiments identified immunopositive sera, anti-KCNQ2N antibodies were purified against the peptide immunogen immobilized on $1 \mathrm{ml}$ columns prepared using SulfoLink (Pierce, Rockford, IL).

Cell culture. Human embryonic kidney (HEK) cells were grown in DMEM/F-12 media supplemented with $10 \%$ fetal bovine serum, penicillin, and streptomycin. Cells were transfected with KCNQ2 or KCNQ3 cDNA, or both, in pcDNA3 expression vectors using FuGene 6 (Roche Molecular Biochemicals, Indianapolis, IN).

Western blot. Mouse brains were homogenized in $0.32 \mathrm{M}$ ice-cold sucrose supplemented with Complete protease inhibitors (Roche Molecular Biochemicals). Crude membranes were prepared by differential centrifugation, resuspended at a concentration of $100 \mathrm{mg}$ of wet tissue/ml in Tris-buffered saline (TBS; $50 \mathrm{~mm}$ Tris and $100 \mathrm{~mm} \mathrm{NaCl}$ ) with protease inhibitors, and stored at $-80^{\circ} \mathrm{C}$ until use. Solubilized proteins from transfected cells were prepared by lysis for $30 \mathrm{~min}$ with ice-cold TBS containing 1\% Triton X-100 and protease inhibitors, followed by centrifugation for $30 \mathrm{~min}$ at $20,000 \times \mathrm{g}$. Supernatants were snap frozen in liquid $\mathrm{N}_{2}$ and stored at $-80^{\circ} \mathrm{C}$ until use. SDS-PAGE, electrotransfer, and Western blotting were performed as described previously (Cooper et al., 2000). To estimate the molecular weights of KCNQ2N-immunoreactive bands on Western blots, lanes were scanned using densitometry software (AlphaInnotech), and positions were compared with molecular weight standards (Precision protein standards; Bio-Rad, Hercules, CA).

Immunohistochemistry. All procedures using animals were in conformity with National Institutes of Health guidelines for the use of laboratory animals and approved by the University of California, San Francisco Committee on Animal Research. Ten adult male C57B6 mice were deeply anesthetized with sodium pentobarbital and perfused via the ascending aorta with $5 \mathrm{ml}$ of PBS followed by $50 \mathrm{ml}$ of cold $4 \%$ paraformaldehyde and $0.1 \%$ glutaraldehyde in $0.1 \mathrm{M}$ sodium phosphate buffer. Brains were removed, post-fixed overnight, and then stored in PBS before sectioning. Floating sections were cut at $20-30 \mathrm{~mm}$ using a vibrating microtome (Leica, Nussloch, Germany).

Immunoperoxidase staining procedures were performed on sections floating in TBS, with additions as noted. Sections were treated with $1 \%$ peroxide to eliminate endogenous peroxidase activity, permeabilized using $0.4 \%$ Triton X-100, blocked using $5 \%$ normal goat serum, $0.1 \%$ bovine serum albumin, and $0.2 \%$ Triton $\mathrm{X}-100$, and then incubated with affinity-purified anti-KCNQ2N antibodies $(0.5 \mu \mathrm{g} / \mathrm{ml})$ for $18-36 \mathrm{hr}$ at $4^{\circ} \mathrm{C}$. After washing (60 min, five changes), sections were incubated in block solution with biotinylated goat anti-rabbit antibodies (1:200; Vector Laboratories, Burlingame, CA) for $2 \mathrm{hr}$ at room temperature. After washing, sections were incubated with ABC solution (Elite kit; Vector Laboratories) for $2 \mathrm{hr}$ at room temperature. Sections were washed in $0.2 \%$ Triton X-100 and TBS for $1 \mathrm{hr}$ and then in $0.1 \mathrm{M}$ Tris. Development of the peroxidase reaction product was performed using $0.5 \mathrm{mg} / \mathrm{ml}$ diaminobenzamine (Sigma, St. Louis, MO) and $0.005 \%$ peroxide. Control experiments using sections processed with omission of the primary antibody, replacement of affinity-purified anti-KCNQ2N antibodies with preimmune sera $(1: 1000)$, or preincubating the antibodies with $1 \mu \mathrm{g} / \mathrm{ml}$ synthetic peptide resulted in loss of immunostaining. Reactions using rabbit anti-VAchT antiserum (1:1000; Roghani et al., 1998) were performed as described above.

For immunofluorescence colocalization experiments, monoclonal antibodies against parvalbumin (1:2000; Sigma) and choline acetyltransferase (1:100; Chemicon, Temecula, CA) were used. Goat anti-rabbit IgG Cy 2 and Goat anti-mouse IgG Cy3 antibodies (Jackson ImmunoResearch, West Grove, PA) were used at 1:500 dilutions. Control experiments showed lack of interaction between the secondary antibodies, species specificity of secondary antibodies, and absence of background staining by secondary antibodies.

Image acquisition and analysis. Light and epifluorescence microscopy were performed using a Nikon (Melville, NY) E800 instrument equipped with a SPOT RT slider digital camera (Diagnostic Instruments). Confocal microscopy was performed using a Bio-Rad MRC 1024 confocal

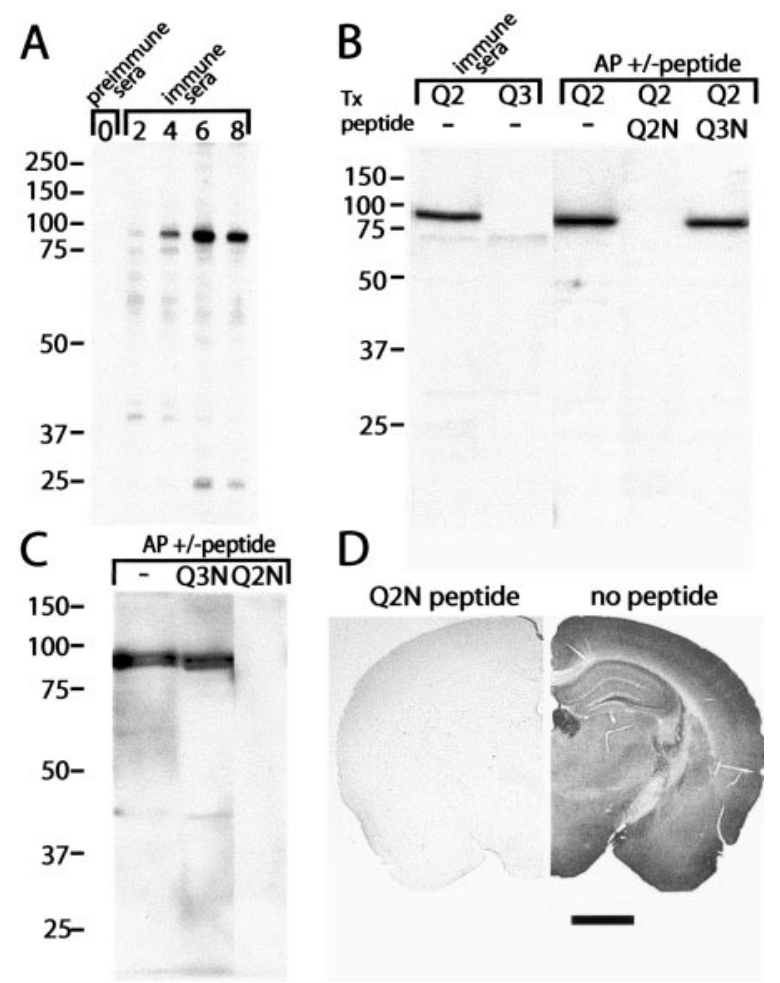

Figure 1. Characterization of anti-KCNQ2N antisera and affinitypurified antibodies. $A$, Western blots using preimmune and immune sera against KCNQ2 expressed in HEK cells. Immunoreactivity against the expressed $\sim 85 \mathrm{kDa}$ KCNQ2 band is absent in preimmune sera and increases progressively during the immunization. $B$, Immune sera detects the HEK-expressed KCNQ2 protein but not KCNQ3 ( $90 \mathrm{kDa}$; data not shown). After affinity purification $(A P)$, an $\sim 70 \mathrm{kDa}$ band background band is eliminated, and recognition of transfected KCNQ2 is abolished by the KCNQ2N peptide but not by a peptide from the KCNQ3 $N$ terminus. $C, \mathrm{KCNQ} 2 \mathrm{~N}$ antibodies detect two bands of $\sim 85$ and $\sim 90 \mathrm{kDa}$ in mouse brain membranes. $D$, Immunoperoxidase staining by KCNQ2N antibodies is abolished by preincubation of the antibodies with the immunogenic peptide. Scale bar, $1 \mathrm{~mm}$. 


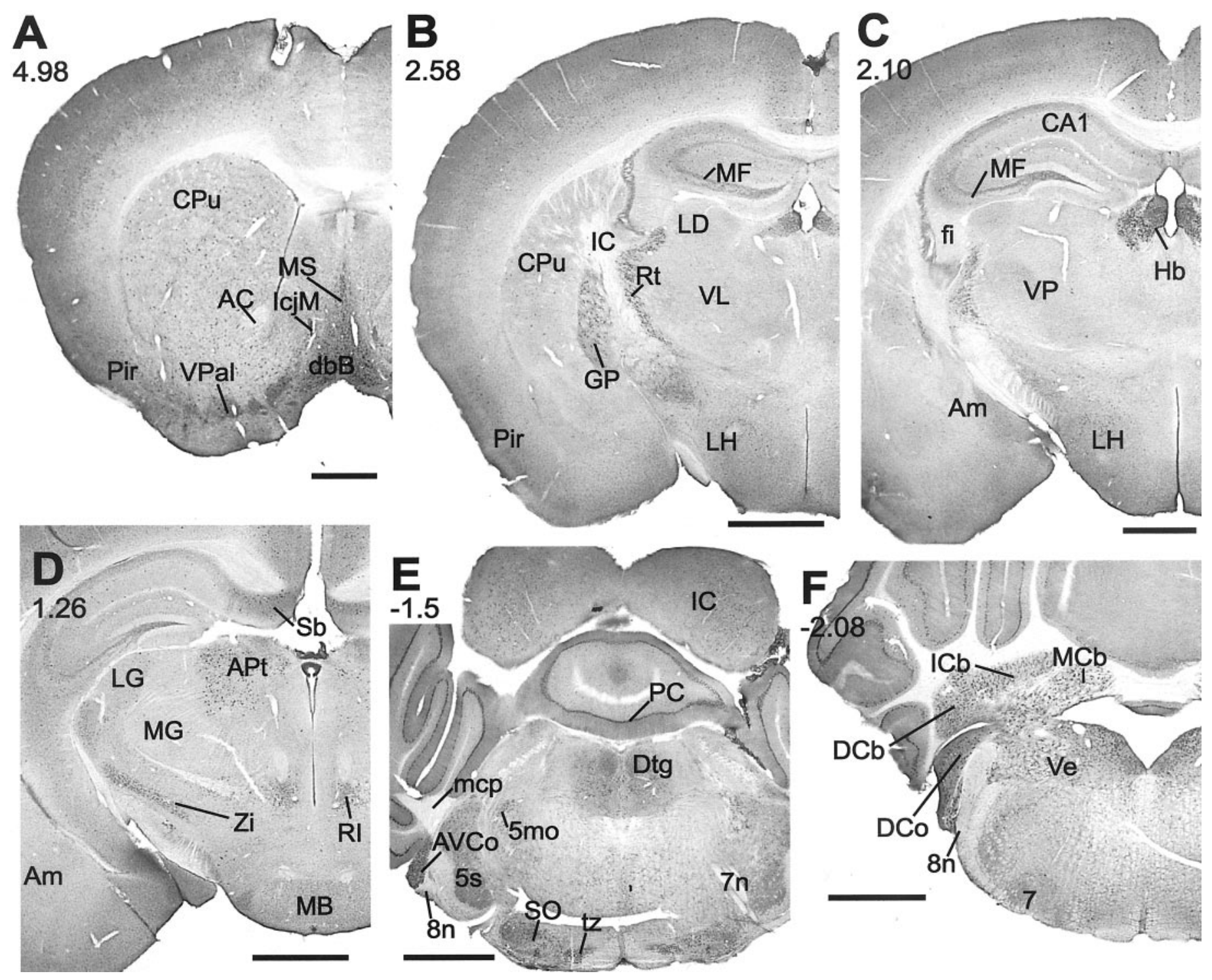

Figure 2. Distribution of KCNQ2N immunoreactivity in the mouse brain. $A$, Regions shown are the piriform cortex (Pir), caudoputamen $(C P u)$, ventral pallidum ( $V P a l)$, island of Calleja major $(I c j M)$, and medial septum $(M S)$ and diagonal band of Broca $(d b B)$. $B$, Section through the globus pallidus $(G P)$, reticular $(R t)$, laterodorsal $(L D)$, and ventrolateral $(V L)$ thalamus, hippocampal mossy fibers $(M F)$, and lateral hypothalamus $(L H)$. $C$, Heavy staining of the mossy fibers and habenula $(H b)$; staining in the fimbria $(f i)$, ventroposterior thalamus $(V P)$, amygdala $(A m)$, and lateral hypothalamus $(L H)$ is lighter or moderate. $D$, Staining in the subiculum $(S b)$, mammillary body $(M B)$, zona incerta $(Z i)$, and anterior pretectal $(A P t)$, lateral geniculate $(L G)$, and rostral interstitial $(R I)$ nuclei. The amygdala and medial geniculate $(M G)$ regions show light staining. $E$, The anteroventral cochlear nucleus $(A V C o)$, sensory division of the trigeminal nucleus $(5 s)$, superior olive $(S O)$, trapezoid body $(t z)$, dorsal tegmentum $(D t g)$, Purkinje cell layer $(P C)$, and inferior colliculus $(I C)$ show moderate to heavy cell body staining. White matter tracts, including the middle cerebellar peduncle $(m c p)$ and facial $(7 n)$ and vestibulocochlear $(8 n)$ nerves are unlabeled. $F$, The vestibular $(V e)$, dorsal cochlear $(D C o)$, and dentate, interposed, and medial cerebellar $(D C b, I c b$, $M C b$ ) nuclei all show heavy staining, but the facial nucleus (7) is unstained. Numbers below the panel letters indicate rostrocaudal positions of the sections according to the atlas of Franklin and Paxinos (1997). Scale bars, $1 \mathrm{~mm}$.

microscope. Figures were prepared using Photoshop (Adobe Systems, Mountain View, CA) and labeled using Adobe Illustrator software, using the stereotactic mouse brain atlas of Franklin and Paxinos (1997) as a guide.

\section{RESULTS}

\section{Characterization of KCNQ2N antibodies}

We raised antibodies against a synthetic peptide corresponding to amino acids $16-37$ within the cytoplasmic $\mathrm{N}$-terminal region of KCNQ2. The chosen sequence is unique to KCNQ2, absolutely conserved in rat, mouse, and human KCNQ2 clones, and is derived from the first exon of the KCNQ2 gene, which is not known to be subject to alternative splicing. We tested immune sera using HEK cells transiently transfected with cDNA encoding an 844-amino acid isoform of human KCNQ2 (Biervert et al., 1998) (Fig. 1 $A, B$ ). Immune (but not preimmune) sera recognized a single predominant band of appropriate size $(\sim 85 \mathrm{kDa})$ in KCNQ2 transfected cells but not in untransfected cells (data not shown) or KCNQ3-transfected cells (Fig. 1B, second lane). Purification of antibodies using the immobilized peptide antigen enhanced the specific detection of the KCNQ2 polypeptide. Preincubation with the KCNQ2N peptide completely and specifically abolished the ability of the affinity-purified antibodies to detect the KCNQ2 band (Fig. 1B, third through fifth lanes). Western blots of adult mouse brain membranes using the affinity-purified 
KCNQ2N antibodies revealed two strong bands of $\sim 85$ and 90 kDa (Fig. 1C), possibly representing known 844- and 872-amino acid "long" forms of KCNQ2 (Biervert et al., 1998; Singh et al., 1998). Lower molecular weight KCNQ2N-immunoreactive bands between $\sim 40$ and $\sim 75 \mathrm{kDa}$ were sometimes detected weakly in Western blots of brain homogenates (data not shown). These lower molecular weight immunoreactive bands, which were completely eliminated when antibodies were preadsorbed with the peptide immunogen, may represent "short" isoforms of KCNQ2N produced by alternative splicing (Nakamura et al., 1998; Pan et al., 2001; Smith et al., 2001). However, they were never prominent, and we have not yet investigated them systematically. The steady-state protein levels of short isoforms of KCNQ2 in adult mouse brain thus appear low compared with those of long forms.

Immunoperoxidase staining of mouse brain tissue sections revealed widespread somatic staining of neurons and more diffuse neuropil staining (discussed below). Staining of neuronal profiles and neuropil was completely abolished by preincubation of the antibodies with the immunogenic peptide (Fig. 1D).

\section{Distribution of KCNQ2N immunoreactivity in mouse brain}

We found extensive KCNQ2N immunoreactivity throughout the mouse brain (Fig. 2). The structures most heavily labeled were neuronal somata in many regions. Neuropil was strongly labeled in cortex, the cerebellar molecular layer, and many subcortical structures. White matter tracts exhibited weak or absent staining.

\section{Olfactory bulb and associated structures}

The olfactory bulb receives input from nasal primary olfactory sensory neurons, processes this input, and relays it to the olfactory cortex (Shepherd and Greer, 1990). The principal neurons, the mitral cells, have their somata in the narrow mitral cell layer and long apical dendrites ending in the glomerular layer, where they receive synaptic inputs from the nasal sensory cells. Several types of local interneurons in these and adjoining external plexiform and internal plexiform layers regulate the responsiveness of mitral cells to their excitatory inputs from the periphery (Shepherd and Greer, 1990). Central modulatory inputs to the bulb include cholinergic and monoaminergic fibers from the diagonal band of Broca, locus ceruleus, and raphe nuclei and a diversity of neuropeptides. KCNQ2N immunostaining in the main olfactory bulb was strongest in the mitral cell and glomerular layers (Fig. $3 A$ ). Somata of the mitral and periglomerular cells, as well as the periglomerular neuropil, were intensively stained. Other layers were lightly stained, except for cell bodies of a few scattered interneurons in the external plexiform layer. The tightly packed somata of the olfactory piriform cortex exhibited moderate staining intensity (Fig. 2A,B).

\section{Septum and diagonal band}

Neurons in the medial septal nucleus (MS) and diagonal band of Broca (dbB) exhibited intense somatic labeling (Figs. $2 A, 3 B$, $4 C, E)$. This region contains GABAergic interneurons, GABAergic projection neurons whose axons innervate the hippocampal formation (see Discussion), as well as cholinergic neurons. Double immunofluorescence staining with parvalbumin (PV) and KCNQ2N indicated that all PV-positive profiles in the MS were also strongly KCNQ2N-immunoreactive (Fig. 4C). Additional PV-negative, KCNQ2N-positive cells were also noted. Staining with choline acetyltransferase (ChAT) revealed that some of these PV-negative, KCNQ2N immunoreactive somata were cho-
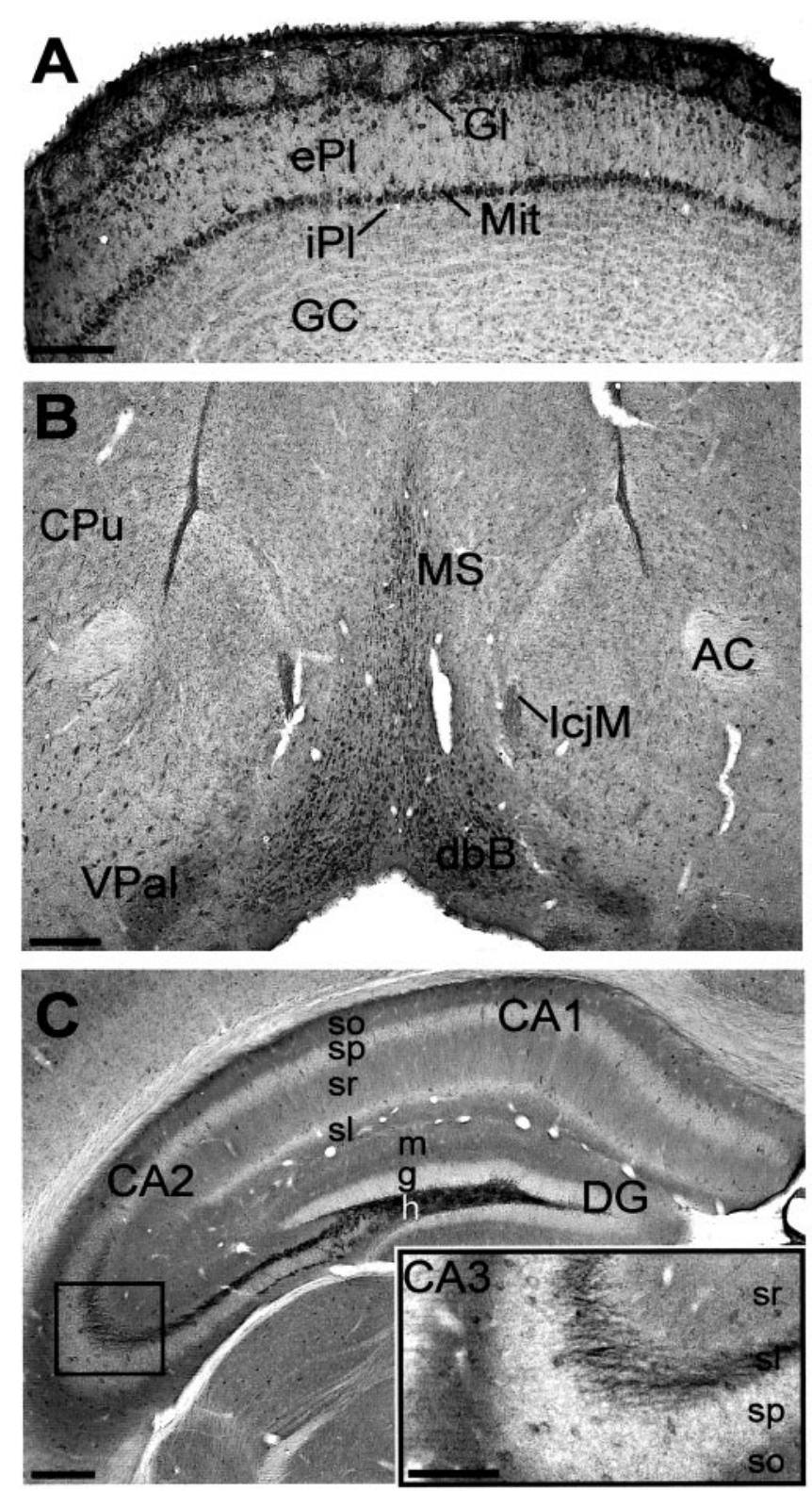

Figure 3. Distribution of KCNQ2N immunoreactivity in the olfactory bulb, ventral forebrain, and hippocampus. $A$, In olfactory bulb, strong neuropil staining is detected in the glomerular layer. Periglomerular cells in the glomerular layer $(G l)$ and mitral cells (Mit) exhibit strong somatic staining. Staining is lighter in the external plexiform $(e P l)$, internal plexiform $(i P l)$, and granule cell $(G C)$ layers. $B$, In the basal forebrain, strong neuropil and somatic staining is apparent in the medial septum $(M S)$, diagonal band of Broca $(d b B)$, ventral pallidum $(V P a l)$, and island of Calleja major $(I c j M)$. The anterior commissure $(A C)$ and caudoputamen $(C P u)$ are indicated. $C$, In the hippocampal formation, neuropil staining is intense along the mossy fiber pathway in the hilus $(h)$ of the dentate gyrus $(D G)$ and stratum lucidum (inset, $s l$ ) of CA3. Moderate neuropil staining is present in the stratum oriens $(s o)$, stratum radiatum $(s r)$, and stratum lacunosum-moleculare $(s l)$ of the hippocampus and the dentate molecular layer $(m)$. In the stratum pyramidale $(s p)$ and stratum granulosum $(g)$, staining is light, except for a few cell bodies (e.g., CA3 inset). Similar profiles are seen in the stratum pyramidale of CA1 and in the dentate granule cell layer (Fig. 4). Scale bars: $A-C, 250 \mu \mathrm{m}$; inset, $125 \mu \mathrm{m}$.

linergic neurons (Fig. 4D); however, many of the large, multipolar ChAT-positive neurons in the MS exhibited low or undetectable KCNQ2N staining (data not shown). Thus, subpopulations of both cholinergic and GABAergic neurons of the MS and $\mathrm{dbB}$ were strongly KCNQ2N-immunoreactive. 

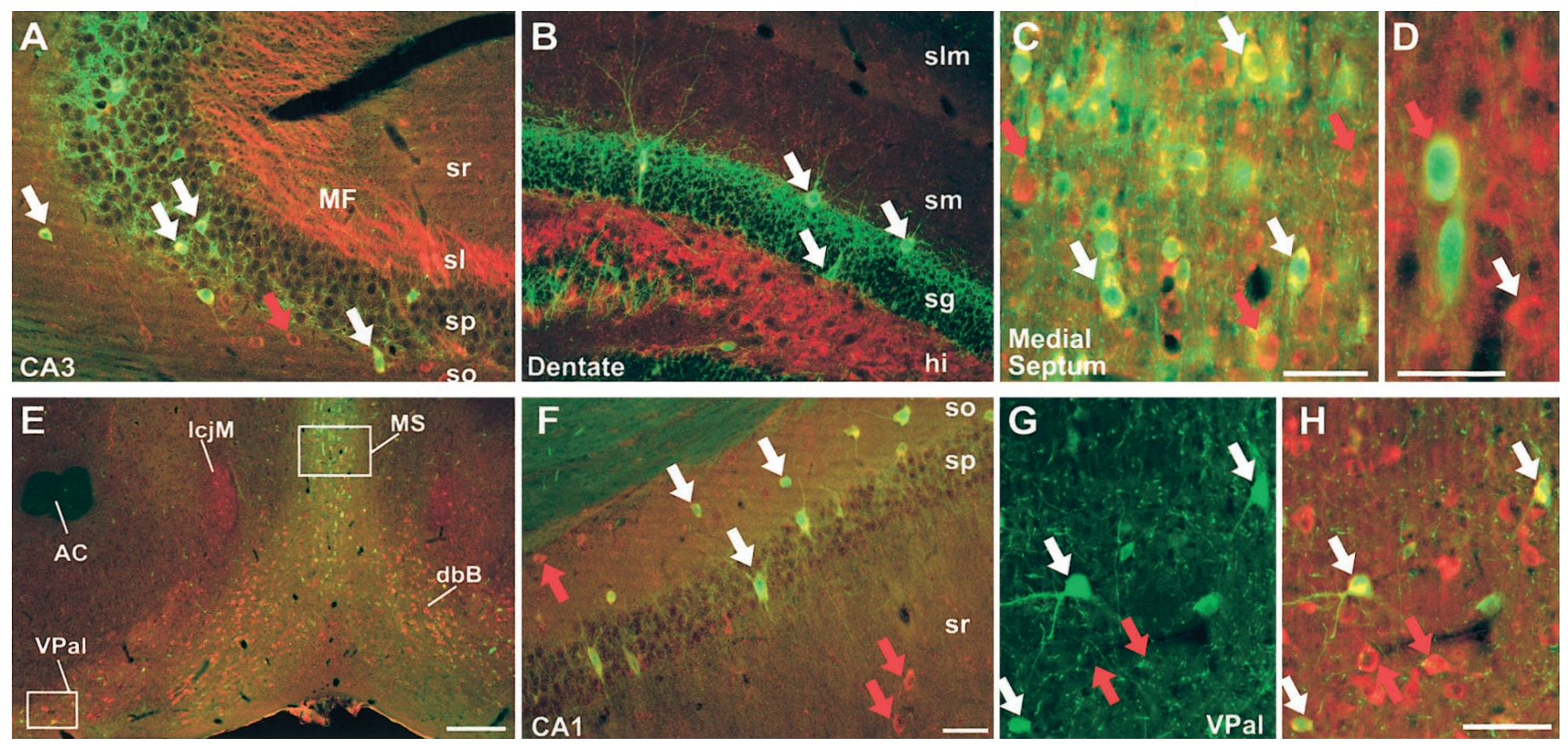

Figure 4. KCNQ2N immunolocalization in the hippocampus and basal forebrain. $A-C, E-G$, Immunofluorescence of sections double-labeled with KCNQ2N (red) and PV (green). A, In CA3, KCNQ2N intensely labels mossy fiber ( $M F$ ) bundles in the stratum lucidum ( $s l$ ) and, less strongly, neuropil of the stratum radiatum ( $s r)$ and stratum oriens $(s o)$. Interneurons in the stratum pyramidale $(s p)$ and oriens are double-labeled by PV and KCNQ2N (white arrows). Additional interneurons in the stratum oriens and radiatum (e.g., red arrow) are KCNQ2N-positive but PV-negative. $B$, In the dentate gyrus, KCNQ2N stains hilar mossy fiber and PV-positive interneurons in the granule cell layer (sg). The stratum lacunosummoleculare $(\mathrm{slm})$, stratum moleculare $(\mathrm{sm})$, and hilus $(\mathrm{hi})$ are indicated. $C$, Detail of $E$. The medial septum contains numerous PV-positive (white arrows) and PV-negative cells labeled with KCNQ2N. D, Colabeling in the medial septum by ChAT (green) and KCNQ2N (red); the red arrow indicates a colabeled cell; the white arrow indicates a KCNQ2N-immunoreactive, ChAT-nonreactive profile. $E$, Low-power view of septal region (as in Fig. $3 B$ ). The anterior commissure $(A C)$ lacks KCNQ2N immunostaining, but the medial septum $(M S)$, island of Calleja major $(I c j M)$, dbB, and ventral pallidum ( $\mathrm{VPal}$ ) show strong somatic and neuropil staining. Boxes indicate regions shown at higher magnification in $C, G$, and $H$. $F$, In CA1, PV-positive (white arrows) and PV-negative (red arrows) interneurons in the stratum oriens, pyramidale, and radiatum show moderate labeling for KCNQ2N. $G, H, \mathrm{PV}$ and KCNQ2N double staining of the ventral pallidum. In $G$, only PV staining is shown; in $H$, KCNQ2N (red) and PV ( green) staining are superimposed. Red arrows indicate position of two of the numerous PV-negative, KCNQ2N-positive cell bodies; white arrows show colabeled cells. Scale bars: $E, 250 \mu \mathrm{m} ; F$ (applies to $A, B$ ), $C, D, G, H, 50 \mu \mathrm{m}$.

In the lateral septal nucleus, moderate neuropil staining was detected, but neurons were unstained (Fig. $5 A$ ). The small, densely packed cells of the triangular septal nucleus, responsible for the strong purinergic input to the habenular region (Sperlágh et al., 1998), exhibited strong staining for KCNQ2N (Fig. 5A, inset).

\section{Basal ganglia}

The basal ganglia include nigrostriatal circuitry that is important for motor control and mesolimbic and ventral pallidal components involved in emotion and reward (Wilson, 1990). Approximately $95 \%$ of rodent caudoputaminal neurons are GABAergic, PV-negative principal cells, the spiny neurons, which project to the globus pallidus and substantia nigra reticulata. The remaining $5 \%$ of caudoputaminal cells are interneurons and include cholinergic neurons and PV- and calbindin-expressing subpopulations of GABAergic cells (Wilson, 1990). Immunoperoxidase staining of the caudoputamen for KCNQ2N revealed light to moderate neuropil staining and conspicuous somatodendritic labeling of a small number of neurons (Fig. 6A). Double staining with KCNQ2N and PV revealed three populations of neurons: those labeled by KCNQ2N alone, PV alone, and both markers (Fig. $6 B-D)$. The somata of neurons labeled by KCNQ2N but not PV appeared larger than those labeled by both KCNQ2N and PV or by PV alone. Because the cholinergic interneurons of the striatum are larger and less numerous than the GABAergic principal cells and interneurons, we suspected that the KCNQ2N-only-labeled cells might include such neurons. Indeed, double immunofluorescence staining for ChAT and KCNQ2N revealed that the larger, most strongly KCNQ2N-immunoreactive cells in the caudoputamen were cholinergic large aspiny neurons with the typical dendritic morphology of these cells (Fig. 6D).

In the globus pallidus, the main target of output from the caudoputamen, both neuropil and neuronal somata exhibited strong KCNQ2N staining (Figs. 2B, 7). The pallidum is reported to consist almost entirely of PV-positive GABAergic neurons (Parent et al., 1996). Unexpectedly, double labeling with PV revealed two populations of KCNQ2N-immunoreactive cells in the globus pallidus: those that expressed both markers (which were far more numerous) and those that expressed KCNQ2N but not PV (which were preferentially located near the periphery of the globus pallidus) (Fig. $7 B, G$ ). In the rodent, cholinergic neurons functionally similar to those of the more ventrally located basal forebrain cholinergic nucleus are found within and at the borders of the globus pallidus and within the internal capsule (Arvidsson et al., 1997). We visualized these "ectopic" cholinergic neurons by immunostaining using a rabbit antibody against VAchT (Fig. 7C). Double labeling with KCNQ2N and ChAT revealed, as expected, that most of the KCNQ2N-labeled cells within the globus pallidus were ChAT-negative. However, a few neurons, preferentially located at the edge of the globus pallidus, were immunoreactive for both KCNQ2N and ChAT (Fig. $7 F$ ). 
Figure 5. KCNQ2N immunostaining of the triangular septal nucleus (TS) and habenula. $A, \mathrm{KCNQ} 2 \mathrm{~N}$ strongly stained somata of tightly packed granular neurons in the triangular nucleus (inset shows higher magnification) and lightly stained the neuropil in the lateral dorsal septal nucleus $(L S D)$. The fibers of the hippocampal commissure $(H C)$ are unstained. $L V$, Lateral ventricle; $C g$, cingulate gyms. $B$, Immunoperoxidase staining of habenula, showing intensely reactive somata in the medial $(M H)$ and lateral $(L H)$ regions. $C$, Colabeling for KCNQ2N (red) and ChAT (green) is exhibited by the cholinergic neurons of the medial habenula but not the neurons of the lateral habenula. The fasciculus retroflexus $(F R)$ the projection pathway of the medial habenula to the interpeduncular nucleus, is strongly ChAT-immunoreactive. $D$, Colabeling with KCNQ2 (red) and PV (green) showing that cholinergic KCNQ2positive medial habenula neurons are PV-negative, as expected. PV staining of the lateral habenula neuropil is intense, and some lateral habenula somata appear to contain both KCNQ2N and PV immunoreactivity. Scale bars: $A, 250 \mu \mathrm{m}$; inset, $B-D, 50 \mu \mathrm{m}$.
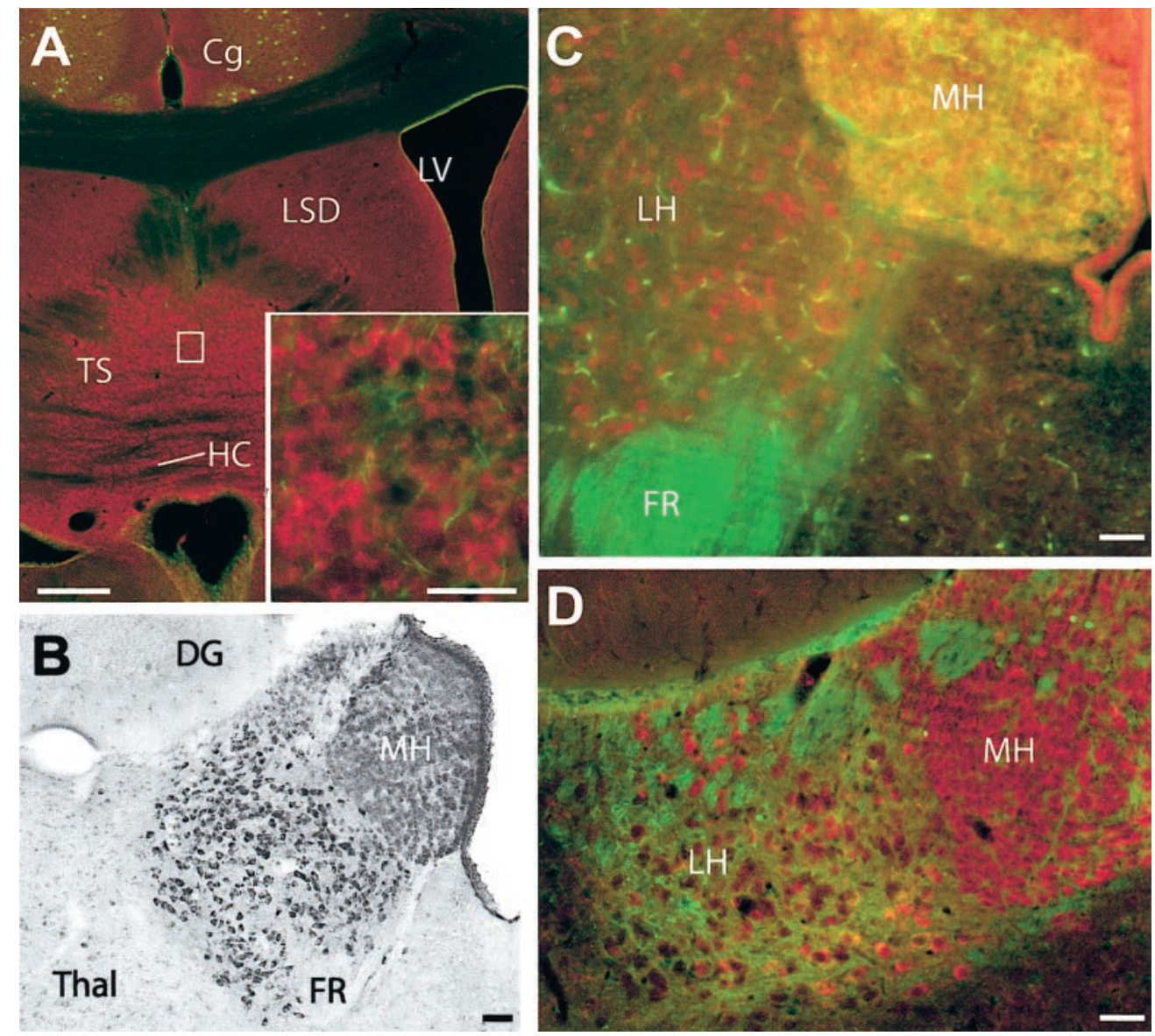

The ventral pallidum and islands of Calleja are important targets of dopaminergic afferents from the ventral tegmental area and are components of mesolimbic circuitry implicated for reward, addiction, emotions, and thought disorders (Missale et al., 1998). These regions exhibited strong neuropil and somatic staining for KCNQ2N (Figs. 3B, 4E, $G, H$ ). Double labeling revealed that some of the KCNQ2N immunoreactive neurons in these regions also expressed PV (Fig. 4G,H).

\section{Amygdala}

Very light neuropil staining was detected throughout the amygdala, and somatic staining was not conspicuous in the region (Fig. $2 C, D)$.

\section{Hippocampal formation}

The hippocampal formation includes the dentate gyrus, hippocampus (CA1, CA2, and CA3), and the subicular complex (Amaral and Witter, 1995). The dentate granule cells receive excitatory inputs on their apical dendrites in the molecular layer; their axons, the mossy fibers, project to form synapses on the apical dendrites of the CA3 pyramidal cells. The CA3 pyramidal cells project in turn to CA1, where they synapse with CA1 pyramidal cells. In addition to this so-called trisynaptic circuit of glutamatergic principal cells, numerous interneurons play critical roles regulating the excitability of the principal cells and helping synchronize pyramidal cell output (Cobb et al., 1995; Freund and Gulyás, 1997). Additional extrinsic cholinergic, monoaminergic, and GABAergic inputs, as well as numerous neuropeptides, modulate the activity of this circuitry (Amaral and Witter, 1995). The most striking feature of the KCNQ2N immunoperoxidase stain- ing pattern in the hippocampal formation was the intense staining of the mossy fiber pathway in the dentate hilus and the stratum lucidum of CA3 (Figs. 3C, 4A). KCNQ2N also stained the neuropil in the dentate molecular layer and stratum oriens, radiatum, and lacunosum-moleculare (Fig. 3C). Scattered throughout all layers in the dentate, hippocampus, and subiculum were neuronal somata stained with moderate intensity (Fig. 3C, inset). Aside from these occasional neuronal profiles, the granule cell and pyramidal cell layers exhibited the lowest intensity of KCNQ2N immunoreactivity in the region. PV marks an important subpopulation of hippocampal interneurons, primarily located within and near the principal cell layers, whose axons arborize widely within the pyramidal layer to form axosomatic synapses with principal cells (Freund and Gulyás, 1997). Double-label immunolabeling revealed that nearly all the KCNQ2N-stained neuronal profiles in the dentate granule cell layer (Fig. $4 B$ ) or pyramidal cell layers of CA3 or CA1 (Fig. $4 A, F$ ) or the subiculum (data not shown) were $\mathrm{PV}$-positive interneurons. In stratum oriens or radiatum, however, most of the scattered KCNQ2N-immunoreactive profiles were not stained for PV.

\section{Habenula and interpeduncular nucleus}

As discussed above, purinergic neurons in the triangular septal nucleus send a massive projection to innervate the medial habenular neurons (Sperlágh et al., 1998), which, in turn, send a cholinergic projection via the myelinated fibers of the fasciculus retroflexus to the interpeduncular nucleus.

The neurons of both the medial and lateral habenula exhibited intense staining for KCNQ2N (Figs. $2 B, C, 5 B-D$ ). The neurons 

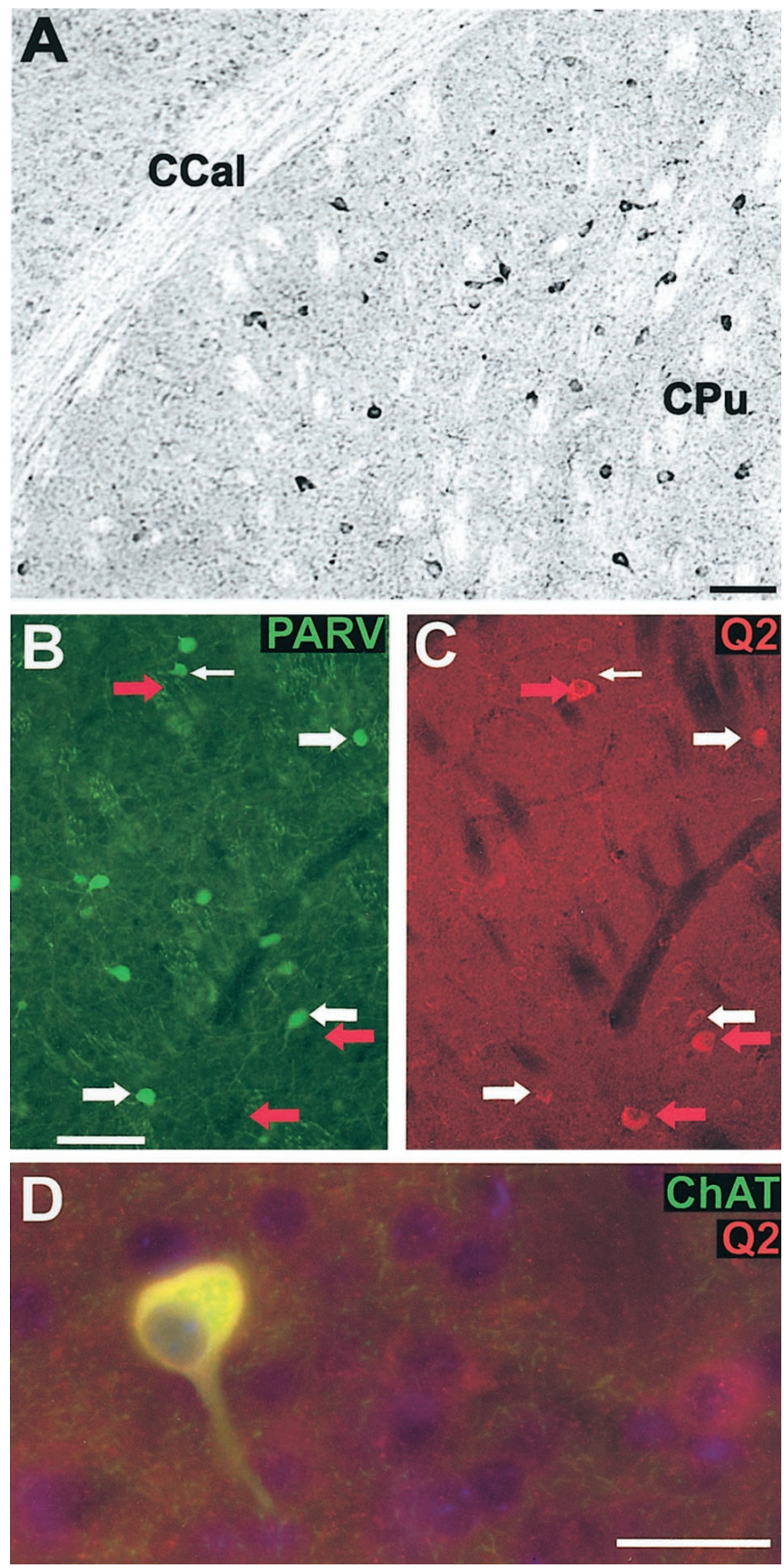

Figure 6. KCNQ2N immunolocalization in the caudoputamen $(\mathrm{CPu}) . A$, Immunoperoxidase stain showing moderate neuropil staining and moderate to heavy staining of somata and proximal dendrites in the $\mathrm{CPu}$, but little staining in the corpus callosum $(\mathrm{CCal}) . \mathrm{B}, \mathrm{C}$, Double immunolabeling for PV (PARV, green) and KCNQ2N (red) in the lateral caudoputamen. Many cells are labeled strongly by PV and weakly by KCNQ2N (e.g., those indicated by wide white arrows). A few, typically larger, cells are labeled by KCNQ2N but not by PV (red arrows) or PV but not KCNQ2N (thin white arrow). D, Double immunolabeling for choline acetyltransferase (green) and KCNQ2N (red) in the caudo putamen. Many cells exhibit staining by both antibodies, but some are stained weakly for KCNQ2 only. Cell nuclei are counterstained with DAPI (blue). Scale bars, $100 \mu \mathrm{m}$.

of the medial habenula and its output pathway, the fasciculus retroflexus, were strongly labeled by ChAT but not by PV. KCNQ2N colabeled the cholinergic neurons in the medial habenula but not the fibers of the fasciculus retroflexus (Fig. $6 C$ ). The
KCNQ2N-labeled neurons of the lateral habenula were unstained by both PV and ChAT but received intense innervation by PVstained fibers (Fig. 5D). The intermediate, lateral, and rostral divisions of the interpeduncular nucleus, targets of cholinergic innervation from the medial habenula via the fasciculus retroflexus, exhibited strong staining of neuronal cell bodies (Fig. 8A).

\section{Thalamus}

Thalamic nuclei represent the final relay point for sensory information of all modalities and also receive reciprocal excitatory inputs from the region of the cerebral cortex to which they project. The activity of thalamic relay neurons and the thalamocortical circuit is strongly influenced by the GABAergic interneurons of the thalamic reticular nucleus. The reticular nucleus is an important target of ascending monoaminergic, cholinergic, and tachykinin inputs that control transitions between sleep and wakefulness (McCormick and Bal, 1997). All of the neurons of the reticular thalamic nucleus were very heavily labeled by KCNQ2N (Figs. 2B, C, 7A,B,D,E). As expected, PV also stained these same neurons strongly. In higher-magnification images, KCNQ2N immunoreactivity had a strong punctate component that appeared in part to be intracellularly localized (Fig. 7E). Although light neuropil staining was present throughout the remaining portions of the thalamus, immunoreactivity of thalamic relay neurons was in general very slight or not apparent. Exceptions to this were the ventral zona incerta (Figs. $2 D, 8 B$ ), the dorsal division of the anterior pretectal nucleus (Fig. 2D), and the rostral interstitial nucleus (Fig. 2D) of the medial longitudinal fasciculus, where many neuronal somata exhibited moderate to heavy KCNQ2N immunoreactivity.

\section{Cerebral cortex}

Neuropil staining for KCNQ2N was present throughout the cerebral cortex, and many neurons exhibited moderate somatic staining (Fig. 2). Examination of sections double-labeled with PV and KCNQ2N and counterstained with 4',6-diamidino-2phenylindole revealed that the intensity of staining of cortical neurons by $\mathrm{KCNQ} 2 \mathrm{~N}$ was very heterogeneous, ranging from inconspicuous to moderate or heavy (data not shown). Highly KCNQ2N-immunoreactive cortical neuronal somata included both PV-positive and -negative cells.

\section{Hypothalamus}

Neurons in the lateral hypothalamic nucleus were stained with moderate intensity (Fig. $2 C$ ). In the mammillary body, neurons and neuropil showed light to moderate staining (Fig. 2D). KCNQ2N staining was absent or light in median, posterior, arcuate, dorsomedian, and ventromedian nuclei.

\section{Midbrain}

KCNQ2N immunoreactivity was strong in the neurons of the medial accessory oculomotor nucleus and magnocellular red nucleus (Fig. $8 A$ ). Strong staining was also seen in the neurons of the substantia nigra reticulata and compacta and the ventral tegmental areas (Fig. 8B,D). A recent immunohistochemical study of KCNQ4 showed strong labeling of the substantia nigra compacta and the ventral tegmental area (Kharkovets et al., 2000). This is intriguing, because KCNQ2 and KCNQ4 are unable to form heteromers when coexpressed in experimental cells (Kubisch et al., 1999), and suggests that two distinct KCNQ channels may be expressed by the same neurons.

In the caudal midbrain, neurons of the central and external 

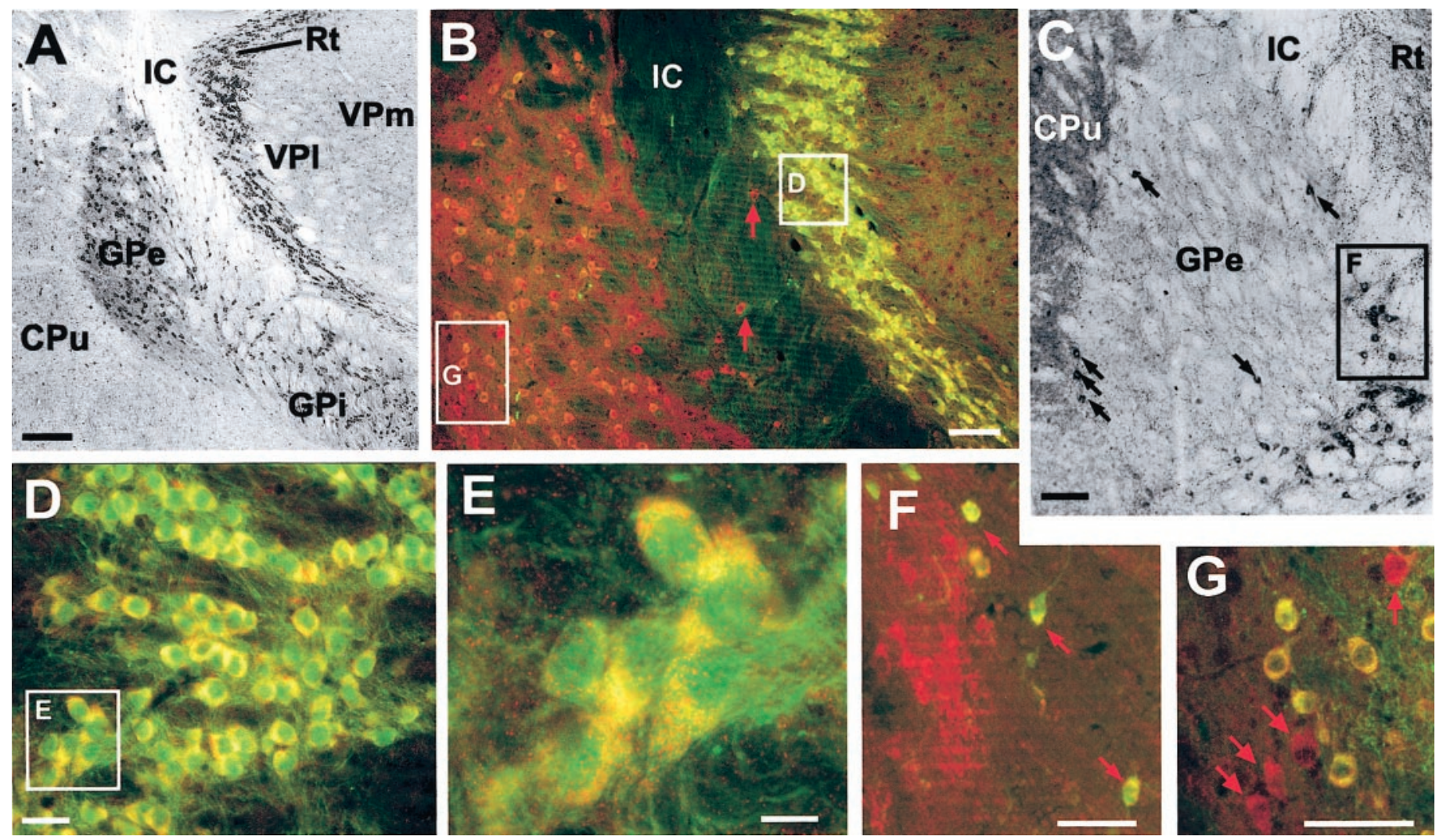

Figure 7. KCNQ2N immunolocalization in the globus pallidus $(G P)$ and nucleus reticularis thalami (Rt). $A$, Immunoperoxidase stain using KCNQ2N antibodies shows strong staining of neuronal profiles in the $C P u$, GP externa and interna (Gpe, Gpi), and Rt, but not in neighboring thalamic relay nuclei, ventroposterior lateral $(\mathrm{VPl})$ and ventroposterior medial $(\mathrm{VPm})$, or the internal capsule $(I C)$. B, Double immunolabeling of a similar section to $A$, using KCNQ2N and PV antibodies. Double-labeled neurons (orange to yellow) are numerous in the nRT and Gpe. In addition, a few neurons (red arrows) in the IC show labeling for KCNQ2N but not PV. $C$, Immunoperoxidase staining with VAchT antibodies reveals the distribution of cholinergic neurons in and near the Gpe. VAchT labels neuropil strongly in the $\mathrm{CPu}$ and moderately in the Gpe and Rt. Intensely labeled neurons are preferentially located at periphery of and ventral to the Gpe. $D$, Higher magnification view of Rt, showing that all PV-labeled neurons are also KCNQ2N labeled. $E$, Detail of $D$, showing punctate appearance of KCNQ2N staining. $F$, Higher magnification view of region near border of IC and ventral border of Gpe (location indicated by box in C), after double immunolabeling with KCNQ2N (red) and ChAT (green). Neuropil and neurons of Gpe are labeled by KCNQ2N only, but a few neurons (arrows) at border are stained by both antibodies. G, Higher magnification views of Gpe double-labeled with PV (green) and KCNQ2N (red). Most cells appear yellow or orange because of colabeling with both markers, but a few preferentially located near periphery of Gpe are labeled by KCNQ2 only (arrows). Scale bars: $A, 250 \mu \mathrm{m} ; B, C, 100 \mu \mathrm{m} ; D, 25 \mu \mathrm{m} ; E, 10 \mu \mathrm{m} ; F, G, 50 \mu \mathrm{m}$.

nucleus of the inferior colliculus were moderately stained, as were neurons of the dorsal tegmental nucleus (Fig. 2E).

\section{Pons and cerebellum}

Neurons of the supratrigeminal nucleus and sensory division of the trigeminal nucleus exhibited moderate KCNQ2N immunoreactivity, but the magnocellular (motor) division of the trigeminal nucleus showed no somatic staining (Fig. $2 E$ ). In the nucleus of the trapezoid body and the superior olive, relay points of the central auditory pathway, somatic staining was strong. Somatic staining was particularly strong in the anteroventral and dorsal divisions of the cochlear nucleus and was moderate in the subnuclei of the vestibular nucleus. Of note, KCNQ4 immunoreactivity is also found in the anteroventral (but not dorsal) cochlear nucleus, superior olive, and inferior colliculus (Kharkovets et al., 2000).

In the cerebellar cortex, the neuropil was moderately stained in the molecular layer and lightly stained in the granule cell layer (Fig. $2 E, F)$. Examination of the cerebellar sections at higher magnification revealed that Purkinje cell somata exhibited strong KCNQ2N immunoreactivity, but, regardless of the orientation of the tissue section, the large proximal dendrites of these cells were not visualized (Fig. 9). This suggested that the neuropil staining in the molecular layer was predominantly attributable to channels located on small distal dendrites or, alternatively, was located on presynaptic fibers or termini (e.g., climbing and parallel fibers). Notable in the granule cell layer was the absence of immunoreactivity associated with the granule cells themselves. However, scattered throughout the thickness of the granular layer were a population of large neurons, stained with moderate intensity on their somata with KCNQ2N. Many of these cells exhibited staining on a single large process oriented toward the Purkinje cell layer (Fig. $9 B$ ). These neurons are likely Golgi cells, on the basis of their morphology and absence of PV immunoreactivity (Fig. 9C; Watanabe et al., 1998). The Golgi and Purkinje neurons both have extensive dendritic arborizations in the cerebellar molecular layer. Trafficking of KCNQ2-containing channels to distal parts of these dendrites could contribute to the strong molecular layer neuropil staining for KCNQ2N. Neurons of the deep cerebellar nuclei also exhibited strong KCNQ2N immunoreactivity (Fig. $2 F$ ).

\section{DISCUSSION}

KCNQ2N immunoreactivity is widely distributed in the mouse brain but is concentrated in particular subsets of neurons in brain 

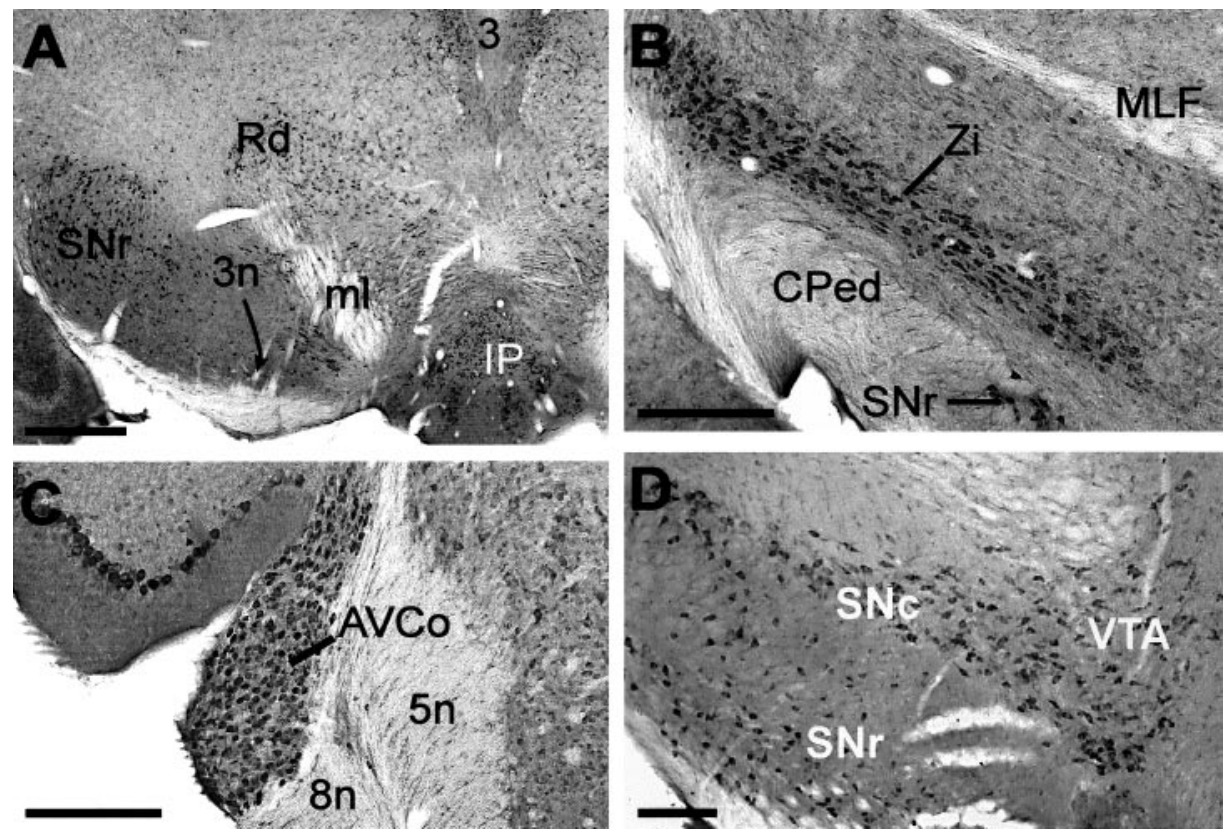

Figure 8. Regions of high KCNQ2N immunoreactivity in thalamus and brainstem. $A$, Ventral midbrain. Staining is detected in the substantia nigra reticulata $(S N r)$ and red $(R d)$, interpeduncular $(I P)$, and oculomotor (3) nuclei. The medial lemniscus $(m l)$ and oculomotor nerve $(3 n)$ are unstained. $B$, Heavy staining is shown in the zona incerta and substantia nigra reticulata, but the cerebral peduncles and medial longitudinal fasciculus (Cped, $M L F$ ) are not stained. $C$, The anteroventral cochlear nucleus $(A V C o$ ) shows somatic staining, but the nearby cochlear nerve and spinal tract of the trigeminal nucleus $(8 n, 5 n)$ are unstained. $D$, Substantia nigra compacta $(S N c)$, substantia nigra reticulata, and ventral tegmental nuclei (VTA) showing somatic staining. Scale bars: $A$, $500 \mu \mathrm{m} ; B-D, 250 \mu \mathrm{m}$. circuits. KCNQ2 is highly expressed in cholinergic and dopaminergic neurons that exert wide-ranging modulatory influences, as well as GABAergic interneurons within several brain regions. Moreover, KCNQ2 appears to be strategically localized in several circuits that control oscillatory neuronal activities. This study provides a road map for additional studies of central KCNQ channels. It highlights the possibility that central KCNQ2containing channels may be important effectors for diverse neurotransmitters (including but not limited to acetylcholine) that modulate the activity of neurons and circuits in which these subunits are expressed.

\section{$M$ current in native neurons}

$I_{\mathrm{M}}$ is one of several currents in rodent superior sympathetic ganglion neurons that are active at rest or are activated between resting and threshold membrane potentials to control excitability and spike frequency (Brown and Adams, 1980; Adams et al., 1986; Jones, 1989). When $I_{M}$ is inhibited by one of several metabotropic receptors, including M1 muscarinic receptors, B2 bradykinin receptors, substance $\mathrm{P}$, and luteinizing hormonereleasing hormone receptors, depolarization is favored, and spike frequency adaptation is reduced. Because peptidergic neurotransmitters in the ganglion have long durations of action and diff use to cells at a distance from their release sites (Jan and Jan, 1982), $I_{\mathrm{M}}$ also serves as a mechanism of sustained, heterosynaptic modulation (Adams and Brown, 1982).

The generalized epileptic phenotype resulting from partial loss-of-function mutations in KCNQ2 or KCNQ3 (Schroeder et al., 1998) is consistent with the hypothesis that these channels function similarly to control excitability in the brain and suggests an additional role in regulation of neuronal synchronization. Thus far, studies of central $I_{M}$ have focused primarily on intrinsic properties of hippocampal CA1 pyramidal cells (Halliwell and Adams, 1982; Malenka et al., 1986; Storm, 1989; Charpak et al., 1990; Charpak and Gähwiler, 1991; Schweitzer et al., 1993; Aiken et al., 1995; Lampe et al., 1997; Madamba et al., 1999; Schweitzer, 2000), and investigations of $I_{M}$ in only a few other central neuronal types have been reported (McCormick and Prince, 1986; Constanti and Sim, 1987). Isolation of
$I_{\mathrm{M}}$ requires an unusual voltage protocol and until recently has been hampered by lack of pharmacological tools. Thus, although many of the neurons that exhibit strong KCNQ2N immunolabeling in our study have received scrutiny by electrophysiologists, it may remain an open question whether $I_{M}$ is a prominent somatic current in these cells. This issue may be addressed by targeted electrophysiological studies of KCNQ2N-immunoreactive neurons, aided by newly developed, selective KCNQ channel blockers and openers (Wang et al., 1998; Rundfeldt, 2000). In some neurons, somatically localized KCNQ2 may be primarily associated with intracellular membranes. These intracellular channels might be destined for trafficking to axonal, dendritic, or perisynaptic sites, or their surface deployment near the soma could be regulated by activity or other factors. Finally, the observation that KCNQ1 forms leak channels when associated with KCNE3 (Schroeder et al., 2000b), highlights the possibility that central channels containing KCNQ2 may exhibit properties different from those of $I_{\mathrm{M}}$.

\section{KCNQ2 is highly expressed in modulatory cholinergic and dopaminergic neurons}

Somatic labeling for KCNQ2N was detected in several cell types within the basal ganglia, including PV-colabeled neurons in the striatum, globus pallidus, and substantia nigra reticulata, dopaminergic neurons of the substantia nigra compacta and ventral tegmental area, and cholinergic neurons in the striatum and near the globus pallidus (Figs. 6-8). The KCNQ2N-immunoreactive cholinergic neurons of the neostriatum, so-called large aspiny neurons, represent only $1-2 \%$ of the striatal neurons but possess dense cholinergic axonal projections throughout the striatum and nucleus accumbens. These projections represent the main cholinergic synapses in the striatum and are thought important in movement disorders and psychiatric disturbances (Wilson, 1990). The dopaminergic neurons of the substantia nigra compacta and ventral tegmental area (Fig. $8 D$ ) provide essential modulatory inputs to their striatal and limbic targets (Haber and Fudge, 1997). 

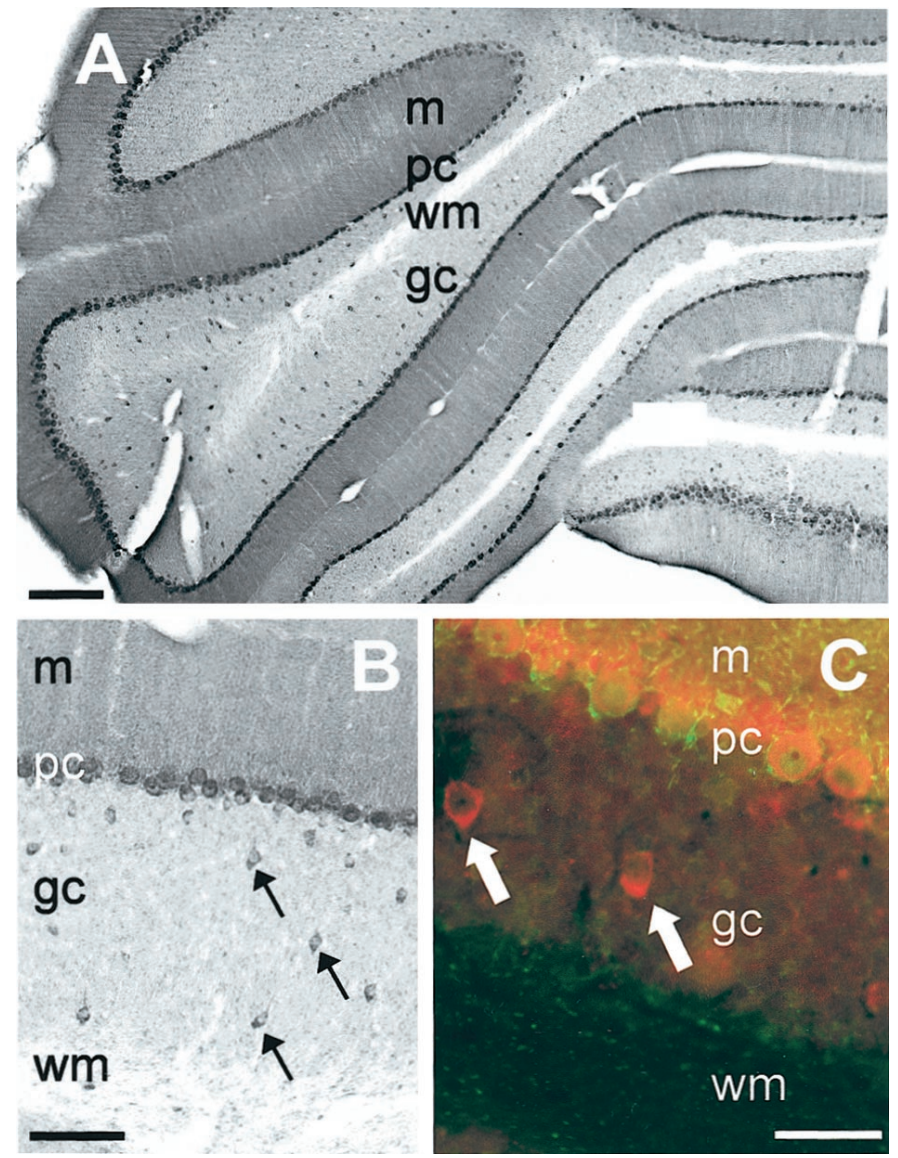

Figure 9. Distribution of KCNQ2N immunoreactivity in the cerebellum. $A$, Immunoperoxidase stain showing strong staining of Purkinje cell ( $p c$ ) somata and molecular level neuropil $(m)$, with light staining of the granule cell layer $(g c)$ and absent staining of deep cerebellar white matter $(w m)$. Scattered cells in the granule cell layer exhibit moderate somatic staining. $B$, Higher-magnification view showing that immunopositive granule cell layer neurons are stained on their somata and apical dendrites. Staining of dendrites of Purkinje cells is not apparent. $C$, Double immunofluorescence image (red, KCNQ2N; green, PV), showing that both markers label Purkinje cells, but KCNQ2N-positive granule cell layer neurons are PV-negative, as expected for Golgi cells. Purkinje cell somata are surrounded by PV-positive axons and termini derived from inhibitory basket and stellate cells whose somata are located in the molecular layer. Scale bars: $A, 250 \mu \mathrm{m} ; B, 100 \mu \mathrm{m} ; C, 50 \mu \mathrm{m}$.

\section{KCNQ2 is strategically localized to septal and hippocampal neurons that regulate hippocampal excitability and synchronization}

We found strong KCNQ2N immunoreactivity on cholinergic and GABAergic neurons in the medial septal region, and on PVpositive and negative interneurons throughout the hippocampal formation (Figs. 3C, 4). These septal and hippocampal neurons are components of a neuronal network regulating the excitability and synchronization of hippocampal pyramidal cells (Cobb et al., 1995; Freund and Gulyás, 1997) (Fig. 10A). Septal cholinergic neurons innervate both cholinergic and GABAergic neurons in the septum and both pyramidal cells and GABAergic interneurons in the hippocampus. Septal GABAergic neurons projecting to the hippocampus selectively innervate hippocampal interneurons (Freund and Antal, 1988). Recent evidence suggests that acetylcholine exerts its most important influence on hippocampal pyramidal neurons via an indirect pathway, by exciting septal GABAergic projection neurons that inhibit hippocampal inter-
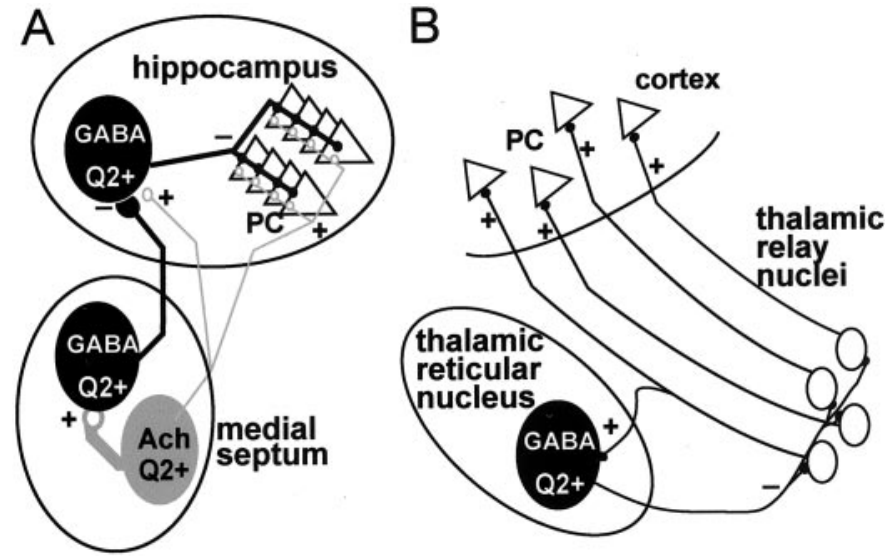

Figure 10. Distribution of KCNQ2N immunoreactivity in septohippocampal and thalamocortical neuronal networks. $A$, In the medial septum, KCNQ2N-immunoreactive $(Q 2+)$ GABAergic projection neurons receive excitatory inputs from nearby $\mathrm{Q} 2+$ cholinergic neurons. Both sets of septal neurons project to the hippocampus. Septal cholinergic fibers innervate all cell types in the hippocampus, but septal GABAergic fibers selectively innervate hippocampal interneurons (some Q2+) that arborize broadly to control activity and synchronization of hippocampal principal cells. $B$, In the thalamocortical network, glutamatergic thalamic relay neurons receive excitatory sensory inputs, and project to pyramidal cells (PC) in the sensory cortex, with collateral output to the $Q 2+$ GABAergic reticular thalamic neurons. Thalamic reticular neurons project back to the relay nuclei, thereby helping synchronize prominent rhythmic firing associated with drowsiness and sleep. Ach, Acetylcholine.

neurons (Tóth et al., 1997; Wu et al., 2000). Because single hippocampal interneurons exert control over many pyramidal cells (Cobb et al., 1995), this network represents a highly amplified mechanism for cholinergic regulation of hippocampal excitability and synchronization (Freund and Gulyás, 1997).

In vivo studies have demonstrated the importance of this septohippocampal network for cognitive function during learning and memory tasks and for hypersynchronization associated with the generation of epileptic seizures. Lesions of the fimbria-fornix, which conveys the axons of the cholinergic and parvalbuminexpressing GABAergic projection neurons from the medial septum to the hippocampus, interfere with learning and memory tasks and with the generation of the theta rhythm in rats. These deficits can be partially reversed by the grafting of cholinergic cells in the hippocampus (Dunnett et al., 1982; Dickinson-Anson et al., 1998). Pharmacological studies argue that the septohippocampal network is a critical site for the effects of muscarinic drugs on learning and memory. Administration of the muscarinic receptor antagonists scopolamine and atropine produces an amnestic syndrome in rodents, primates, and humans (Deutsch and Rocklin, 1967; Rusted, 1988; Rusted and Warburton, 1988; Rupniak et al., 1989). In contrast, muscarinic agonists infused into the medial septum elicit continuous hippocampal theta and facilitate learning and memory in both young and aged rats (Givens and Olton, 1990; Monmaur and Breton, 1991; Lawson and Bland, 1993; Markowska et al., 1995). In rodents, localized septal infusion of the muscarinic agonist carbachol has been shown to reverse the amnesia produced by systemic administration of scopolamine (Givens and Olton, 1995). In contrast, administration of high doses of pilocarpine, another muscarinic agonist, produces status epilepticus in rodents and a chronic, epileptic phenotype that serves as a leading experimental model of human temporal lobe epilepsy (Cavalheiro et al., 1996). 


\section{KCNQ2 expression in reticular neurons that regulate thalamocortical oscillations}

Distinct patterns of rhythmic activity in thalamocortical circuits are correlated with levels of sleep and arousal (Steriade and Llinás, 1988). Abnormal rhythmic activity in this circuit is likely important in many forms of generalized epilepsy (McCormick and Contreras, 2001). GABAergic neurons in the thalamic reticular nucleus regulate thalamocortical oscillations and help synchronize the firing of thalamic relay neurons (McCormick and Bal, 1997) (Fig. 10B). Reticular neurons exhibited strong KCNQ2N immunoreactivity (Fig. 7). Activation of serotonergic, adrenergic, metabotropic glutamate, or tachykinin receptors on reticular nucleus neurons causes slow membrane depolarization as the result of inhibition of a linear leak-type potassium current (McCormick and Wang, 1991; Cox et al., 1995), bringing about a transition from bursting to relay-type firing patterns (McCormick and Bal, 1997). Although purely speculative, it would be interesting to test whether this potassium current arises from KCNQ2 and auxiliary subunits in a manner analogous to the interactions of KCNQ1 and KCNE3.

\section{REFERENCES}

Adams PR, Brown DA (1982) Synaptic inhibition of the M-current: slow excitatory post-synaptic potential mechanism in bullfrog sympathetic neurones. J Physiol (Lond) 332:263-272.

Adams PR, Jones SW, Pennefather P, Brown DA, Koch C, Lancaster B (1986) Slow synaptic transmission in frog sympathetic ganglia. J Exp Biol 124:259-285.

Aiken SP, Lampe BJ, Murphy PA, Brown BS (1995) Reduction of spike frequency adaptation and blockade of $\mathrm{M}$-current in rat $\mathrm{CA}_{1}$ pyramidal neurones by linopirdine (DuP 996) a neurotransmitter release enhancer. Br J Pharmacol 115:1163-1168.

Amaral DG, Witter MP (1995) Hippocampal formation. In: The rat nervous system (Paxinos G, ed), pp 433-495. San Diego: Academic.

Arvidsson U, Riedl M, Elde R, Meister B (1997) Vesicular acetylcholine transporter (VAChT) protein: a novel and unique marker for cholinergic neurons in the central and peripheral nervous systems. J Comp Neurol 378:454-467.

Biervert C, Schroeder BC, Kubisch C, Berkovic SF, Propping P, Jentsch TJ, Steinlein OK (1998) A potassium channel mutation in neonatal human epilepsy. Science 279:403-406.

Brown DA, Adams PR (1980) Muscarinic suppression of a novel voltage-sensitive $\mathrm{K}+$ current in a vertebrate neuron. Nature 283:673-676

Cavalheiro EA, Santos NF, Priel MR (1996) The pilocarpine model of epilepsy in mice. Epilepsia 37:1015-1019.

Charpak S, Gähwiler BH (1991) Glutamate mediates a slow synaptic response in hippocampal slice cultures. Proc R Soc Lond B Biol Sci 243:221-226.

Charpak S, Gähwiler BH, Do KQ, Knöpfel T (1990) Potassium conductances in hippocampal neurons blocked by excitatory amino-acid transmitters. Nature 347:765-767.

Cobb SR, Buhl EH, Halasy K, Paulsen O, Somogyi P (1995) Synchronization of neuronal activity in hippocampus by individual GABAergic interneurons. Nature 378:75-78.

Constanti A, Sim JA (1987) Calcium-dependent potassium conductance in guinea-pig olfactory cortex neurones in vitro. J Physiol (Lond) 387:173-194.

Cooper EC, Aldape KD, Abosch A, Barbaro NM, Berger MS, Peacock WS, Jan YN, Jan LY (2000) Colocalization and coassembly of two human brain M-type potassium channel subunits that are mutated in epilepsy. Proc Natl Acad Sci USA 97:4914-4919.

Cox CL, Huguenard JR, Prince DA (1995) Cholecystokinin depolarizes rat thalamic reticular neurons by suppressing a $\mathrm{K}+$ conductance. J Neurophysiol 74:990-1000.

Deutsch JA, Rocklin KW (1967) Amnesia induced by scopolamine and its temporal variations. Nature 216:89-90.

Dickinson-Anson H, Aubert I, Gage FH, Fisher LJ (1998) Hippocampal grafts of acetylcholine-producing cells are sufficient to improve behavioural performance following a unilateral fimbria-fornix lesion. Neuroscience 84:771-781.

Dunnett SB, Gage FH, Björklund A, Stenevi U, Low WC, Iversen SD (1982) Hippocampal deafferentation: transplant-derived reinnervation and functional recovery. Scand J Psychol [Suppl] 1:104-111.

Franklin KB, Paxinos G (1997) The mouse brain in stereotaxic coordinates. San Diego: Academic.
Freund TF, Antal M (1988) GABA-containing neurons in the septum control inhibitory interneurons in the hippocampus. Nature 336:170-173.

Freund TF, Gulyás AI (1997) Inhibitory control of GABAergic interneurons in the hippocampus. Can J Physiol Pharmacol 75:479-487.

Givens B, Olton DS (1995) Bidirectional modulation of scopolamineinduced working memory impairments by muscarinic activation of the medial septal area. Neurobiol Learn Mem 63:269-276.

Givens BS, Olton DS (1990) Cholinergic and GABAergic modulation of medial septal area: effect on working memory. Behav Neurosci 104:849-855.

Haber SN, Fudge JL (1997) The primate substantia nigra and VTA: integrative circuitry and function. Crit Rev Neurobiol 11:323-342.

Halliwell JV, Adams PR (1982) Voltage-clamp analysis of muscarinic excitation in hippocampal neurons. Brain Res 250:71-92.

Jan LY, Jan YN (1982) Peptidergic transmission in sympathetic ganglia of the frog. J Physiol (Lond) 327:219-246.

Jones SW (1989) On the resting potential of isolated frog sympathetic neurons. Neuron 3:153-161.

Kharkovets T, Hardelin JP, Safieddine S, Schweizer M, El-Amraoui A, Petit C, Jentsch TJ (2000) KCNQ4, a K+ channel mutated in a form of dominant deafness, is expressed in the inner ear and the central auditory pathway. Proc Natl Acad Sci USA 97:4333-4338.

Kubisch C, Schroeder BC, Friedrich T, Lütjohann B, El-Amraoui A, Marlin S, Petit C, Jentsch TJ (1999) KCNQ4, a novel potassium channel expressed in sensory outer hair cells, is mutated in dominant deafness. Cell 96:437-446.

Lampe BJ, Gaskill JL, Keim SC, Brown BS (1997) Linopirdine reduces stimulus intensity threshold for induction of long-term potentiation in the Schaffer collateral/CA1 pathway in rat hippocampal slices. Neurosci Lett 222:135-137.

Lawson VH, Bland BH (1993) The role of the septohippocampal pathway in the regulation of hippocampal field activity and behavior: analysis by the intraseptal microinf usion of carbachol, atropine, and procaine. Exp Neurol 120:132-144.

Madamba SG, Schweitzer P, Siggins GR (1999) Dynorphin selectively augments the M-current in hippocampal CA1 neurons by an opiate receptor mechanism. J Neurophysiol 82:1768-1775.

Malenka RC, Madison DV, Andrade R, Nicoll RA (1986) Phorbol esters mimic some cholinergic actions in hippocampal pyramidal neurons. J Neurosci 6:475-480.

Markowska AL, Olton DS, Givens B (1995) Cholinergic manipulations in the medial septal area: age-related effects on working memory and hippocampal electrophysiology. J Neurosci 15:2063-2073.

McCormick DA, Bal T (1997) Sleep and arousal: thalamocortical mechanisms. Annu Rev Neurosci 20:185-215.

McCormick DA, Contreras D (2001) On the cellular and network bases of epileptic seizures. Annu Rev Physiol 63:815-846.

McCormick DA, Prince DA (1986) Mechanisms of action of acetylcholine in the guinea-pig cerebral cortex in vitro. J Physiol (Lond) 375:169-194.

McCormick DA, Wang Z (1991) Serotonin and noradrenaline excite GABAergic neurones of the guinea-pig and cat nucleus reticularis thalami. J Physiol (Lond) 442:235-255.

Missale C, Nash SR, Robinson SW, Jaber M, Caron MG (1998) Dopamine receptors: from structure to function. Physiol Rev 78:189-225.

Monmaur P, Breton P (1991) Elicitation of hippocampal theta by intraseptal carbachol injection in freely moving rats. Brain Res 544:150-155.

Nakamura M, Watanabe H, Kubo Y, Yokoyama M, Matsumoto T, Sasai H, Nishi Y (1998) KQT2, a new putative potassium channel family produced by alternative splicing. Isolation, genomic structure, and alternative splicing of the putative potassium channels. Receptors Channels 5:255-271.

Pan Z, Selyanko AA, Hadley JK, Brown DA, Dixon JE, McKinnon D (2001) Alternative splicing of KCNQ2 potassium channel transcripts contributes to the functional diversity of M-currents. J Physiol (Lond) 531:347-358.

Parent A, Fortin M, Côté PY, Cicchetti F (1996) Calcium-binding proteins in primate basal ganglia. Neurosci Res 25:309-334.

Roghani A, Shirzadi A, Butcher LL, Edwards RH (1998) Distribution of the vesicular transporter for acetylcholine in the rat central nervous system. Neuroscience 82:1195-1212.

Rundfeldt C (2000) The new anticonvulsant retigabine (D-23129) activates $\mathrm{M}$-currents in Chinese hamster ovary-cells transfected with human KCNQ2/3 subunits. Neurosci Lett 282:73-76.

Rupniak NM, Steventon MJ, Field MJ, Jennings CA, Iversen SD (1989) Comparison of the effects of four cholinomimetic agents on cognition in primates following disruption by scopolamine or by lists of objects. Psychopharmacology 99:189-195.

Rusted JM (1988) Dissociative effects of scopolamine on working memory in healthy young volunteers. Psychopharmacology 96:487-492.

Rusted JM, Warburton DM (1988) The effects of scopolamine on working memory in healthy young volunteers. Psychopharmacology 96:145-152.

Sanguinetti MC, Curran ME, Zou A, Shen J, Spector PS, Atkinson DL, 
Keating MT (1996) Coassembly of K(V)LQT1 and minK (IsK) proteins to form cardiac I(Ks) potassium channel. Nature 384:80-83.

Schroeder BC, Kubisch C, Stein V, Jentsch TJ (1998) Moderate loss of function of cyclic-AMP-modulated KCNQ2/KCNQ3 K channels causes epilepsy. Nature 396:687-690.

Schroeder BC, Hechenberger M, Weinreich F, Kubisch C, Jentsch TJ (2000a) KCNQ5, a novel potassium channel broadly expressed in brain, mediates M-type currents. J Biol Chem 275:24089-24095.

Schroeder BC, Waldegger S, Fehr S, Bleich M, Warth R, Greger R, Jentsch TJ (2000b) A constitutively open potassium channel formed by KCNQ1 and KCNE3. Nature 403:196-199.

Schweitzer P (2000) Cannabinoids decrease the $\mathrm{K}(+)$ M-current in hippocampal CA1 neurons. J Neurosci 20:51-58.

Schweitzer P, Madamba S, Champagnat J, Siggins GR (1993) Somatostatin inhibition of hippocampal CA1 pyramidal neurons: mediation by arachidonic acid and its metabolites. J Neurosci 13:2033-2049.

Shepherd GM, Greer CA (1990) Olfactory bulb. In: Synaptic organization of the brain (Shepherd GM, ed), pp 133-169. New York: Oxford UP.

Singh NA, Charlier C, Stauffer D, DuPont BR, Leach RJ, Melis R, Ronen GM, Bjerre I, Quattlebaum T, Murphy JV, McHarg ML, Gagnon D, Rosales TO, Peiffer A, Anderson VE, Leppert M (1998) A novel potassium channel gene, KCNQ2, is mutated in an inherited epilepsy of newborns. Nat Genet 18:25-29.

Smith JS, Iannotti CA, Dargis P, Christian EP, Aiyar J (2001) Differential expression of KCNQ2 splice variants: Implications to M current function during neuronal development. J Neurosci 21:1096-1103.

Sperlágh B, Maglóczky Z, Vizi ES, Freund TF (1998) The triangular septal nucleus as the major source of ATP release in the rat habenula: a combined neurochemical and morphological study. Neuroscience 86:1195-1207.

Steriade M, Llinás RR (1988) The functional states of the thalamus and the associated neuronal interplay. Physiol Rev 68:649-742.

Storm JF (1989) An after-hyperpolarization of medium duration in rat hippocampal pyramidal cells. J Physiol (Lond) 409:171-190.

Tóth K, Freund TF, Miles R (1997) Disinhibition of rat hippocampal pyramidal cells by GABAergic afferents from the septum. J Physiol (Lond) 500:463-474.

Wang H-S, Pan Z, Shi W, Brown BS, Wymore RS, Cohen IS, Dixon JE, McKinnon D (1998) KCNQ2 and KCNQ3 potassium channel subunits: molecular correlates of the M-channel. Science 282:1890-1893.

Wang Q, Curran ME, Splawski I, Burn TC, Millholland JM, VanRaay TJ, Shen J, Timothy KW, Vincent GM, de Jager T, Schwartz PJ, Toubin JA, Moss AJ, Atkinson DL, Landes GM, Connors TD, Keating MT (1996) Positional cloning of a novel potassium channel gene: KVLQT1 mutations cause cardiac arrhythmias. Nat Genet 12:17-23.

Watanabe D, Inokawa H, Hashimoto K, Suzuki N, Kano M, Shigemoto R, Hirano T, Toyama K, Kaneko S, Yokoi M, Moriyoshi K, Suzuki M, Kobayashi K, Nagatsu T, Kreitman RJ, Pastan I, Nakanishi S (1998) Ablation of cerebellar Golgi cells disrupts synaptic integration involving GABA inhibition and NMDA receptor activation in motor coordination. Cell 95:17-27.

Wilson CJ (1990) Basal ganglia. In: Synaptic organization of the brain (Shepherd GM, ed), pp 279-316. New York: Oxford UP.

Wu M, Shanabrough M, Leranth C, Alreja M (2000) Cholinergic excitation of septohippocampal GABA but not cholinergic neurons: implications for learning and memory. J Neurosci 20:3900-3908. 